\title{
The Inverse Sturm-Liouville Problem and the Rayleigh-Ritz Method
}

\author{
By Ole H. Hald
}

\begin{abstract}
In this paper we present an algorithm for solving the inverse Sturm-Liouville problem with symmetric potential and Dirichlet boundary conditions. The algorithm is based on the Rayleigh-Ritz method for calculating the eigenvalues of a two point boundary value problem, and reduces the inverse problem for the differential equation to a nonstandard discrete inverse eigenvalue problem. It is proved that the solution of the discrete problem converges to the solution of the continuous problem. Finally, we establish the stability of the method and give numerical examples.
\end{abstract}

Introduction. In this paper we will present a numerical method for solving the inverse Sturm-Liouville problem. We will prove that the algorithm converges, and will give numerical results.

The inverse Sturm-Liouville problem is primarily a model problem. In essence it amounts to determining the density of a vibrating string from its fundamental tone and overtones, see Borg [4, p. 83] and Krein [19]. Similar, but more complicated, problems occur in geophysics and engineering. One of the fundamental problems in geophysics is to determine the variation of the density within the earth from the eigenfrequencies of the earth, see Backus and Gilbert [2]. These data can be obtained from seismograph recordings after major earthquakes. In mechanical engineering inverse problems arise in the design of driving shafts. Here it is important that the eigenfrequencies of the shaft do not coincide with the contemplated rotational frequency, see Niordson [25].

The basic paper on the mathematical aspects of the inverse Sturm-Liouville problem is that of Borg [4]. Later more elegant uniqueness proofs have been given by Marčenko [23], Levinson [21], and Hochstadt [15]. Alternate constructive methods have been suggested by Krein [18], Gel'fand and Levitan [10], Levitan [22], Niordson [25], Barcilon [3], Friedland [8], Hochstadt [16], and Hald [13].

The algorithm presented here is based on the classical Rayleigh-Ritz method. The idea is to expand the potential and the eigenfunctions in Fourier series, truncate the series and reduce the problem to a nonstandard finite dimensional inverse eigenvalue problem. The existence of solutions to this problem is easily proved by the contraction mapping theorem. The difficult step is to show that the solutions of the finite dimensional problems converge toward the correct solution of the continuous problem. This crucial point is missing in the discrete algorithms presented by Gantmacher and Krein [9], Anderson [1], Hald [12] and Morel [24].

Received November 8, 1976; revised September 22, 1977. AMS (MOS) subject classifications (1970). Primary 65L15.

Copyright @ 1978, American Mathematical Society 
The problem studied in this paper is a special case of the inverse Sturm-Liouville problem. First, we only consider Dirichlet boundary conditions. Secondly, we assume that the potential is symmetric around the midpoint of the interval; and finally, in order to prove that the method converges, the potential must be sufficiently small in norm. Alternative methods for more general problems have already been proposed by Hald [13] and Hochstadt [16]. Presumably, the basic idea in this paper can be extended to some of these cases, but it seems unlikely that the third restriction can be removed.

1. The Rayleigh-Ritz Method and an Inverse Matrix Problem. In this section we will derive the algorithm and indicate why it works. We consider the Sturm-Liouville problem

$$
-y^{\prime \prime}+q(x) y=\lambda y
$$

on the interval $0 \leqslant x \leqslant \pi$, with the Dirichlet boundary conditions

$$
y(0)=y(\pi)=0 \text {. }
$$

Here $q$ is a real, square-integrable function. This problem has an infinite number of eigenvalues $\lambda_{n}$ with $\lambda_{n} \rightarrow \infty$, and a corresponding set of eigenfunctions $y_{n}(x)$, see [6, pp. 189-201]. The inverse Sturm-Liouville problem amounts to the reconstruction of the function $q$ from $\lambda_{1}, \lambda_{2}, \ldots$ Borg has shown that the eigenvalues determine the potential uniquely provided it satisfies the symmetry condition

$$
q(x)=q(\pi-x)
$$

for almost all $x$ in $[0, \pi]$, see $[4$, p. 69]. We will therefore construct our solution such that Eq. (1.3) is fulfilled.

By multiplying Eq. (1.1) with $y(x)$, integrating by parts and using the boundary conditions (1.2), we see that the eigenfunctions $y_{n}$ are the stationary points of the Rayleigh quotient

$$
R[y]=\frac{\int_{0}^{\pi} y^{\prime 2}+q y^{2}}{\int_{0}^{\pi} y^{2}},
$$

cf. Courant-Hilbert [7, p. 402]. To compute the critical points we take a classical approach and use trigonometric polynomials as trial functions. If $y=\Sigma_{1}^{n} w_{j} \sin j x$, then the Rayleigh quotient reduces to the quadratic form

$$
R[w]=w^{T} A w / w^{T} w .
$$

Here $w^{T}=\left(w_{1}, \ldots, w_{n}\right)$ and the elements of the symmetric $n \times n$ matrix $A=$ $\left(a_{j l}\right)$ are given by

$$
a_{j l}=j^{2} \delta_{j l}+\frac{2}{\pi} \int_{0}^{\pi} q(x) \sin j x \sin l x d x
$$

If the potential is constant, then $A$ is a diagonal matrix and the differential equations (1.1), (1.2) have the same eigenvalues as $A$. Otherwise, we extend the potential 
as an even, $2 \pi$ periodic function and represent it by its Fourier series

$$
q(x)=\alpha_{0}+2 \sum_{k=1}^{m} \alpha_{k} \cos 2 k x,
$$

where $m \leqslant \infty$. Here all odd Fourier coefficients vanish because of the symmetry condition (1.3). Borg has shown that the eigenvalues of the differential equation have the asymptotic expansion $\lambda_{j}=j^{2}+\alpha_{0}+o(1)$, see [4, p. 11]. In the inverse problem the $\lambda_{j}$ are given; and we may therefore assume that $\alpha_{0}$ vanishes.

To motivate our algorithm for the inverse Sturm-Liouville problem we consider a typical case, say $n=m=6$. By inserting (1.5) in Eq. (1.4), we obtain

$$
A=\left[\begin{array}{ccc}
1-\alpha_{1} & \alpha_{1}-\alpha_{2} & \alpha_{2}-\alpha_{3} \\
2^{2}-\alpha_{2} & \alpha_{1}-\alpha_{3} & \alpha_{2}-\alpha_{4} \\
\alpha_{1}-\alpha_{2} & 3^{2}-\alpha_{3} & \alpha_{1}-\alpha_{4} \\
\alpha_{2}-\alpha_{3} & 4^{2}-\alpha_{4} & \alpha_{1}-\alpha_{5} \\
\alpha_{2}-\alpha_{4}-\alpha_{4} & 5^{2}-\alpha_{5} \\
\alpha_{2} & \alpha_{1}-\alpha_{5} & 6^{2}-\alpha_{6}
\end{array}\right]
$$

In the direct problem the eigenvalues of $A$ approximate the eigenvalues $\lambda_{j}$ of the differential equation (1.1). In the inverse problem $\lambda_{j}$ are given, and we will determine $\alpha_{k}$ such that $\lambda_{j}$ are the eigenvalues of $A$. Thus, we expect that the eigenvalues of the differential equation (1.1) with the potential (1.5) are close to $\lambda_{j}$ and hope that the potentials converge toward the solution of the inverse Sturm-Liouville problem as $n$ and $m$ increase. Observe here that if $m<<n$, then $A$ will be a band matrix; whereas if $n$ is fixed, then all matrices with $m \geqslant n$ are identical.

We can now formulate our inverse matrix problem. Let $D=\operatorname{diag}\left(1,2^{2}, 3^{2}, \ldots\right)$. Find a matrix $E$ such that $\lambda_{j}$ are the eigenvalues of $A=D+E$. Here the off-diagonal elements of $E$ depend on the diagonal elements. This problem has much in common with the so-called additive inverse eigenvalue problem which has been studied extensively; for recent results see Morel [24] and Friedland [8] ; for older results see the survey by Hald [12].

Before presenting our algorithm we observe that the eigenvalue problem for the matrix $A$ can be reduced to the eigenvalue problems for $A^{I}$ and $A^{I I}$. If $m \geqslant n=6$, then

$$
A^{I}=\left[\begin{array}{ccc}
1-\alpha_{1} & \alpha_{1}-\alpha_{2} & \alpha_{2}-\alpha_{3} \\
\alpha_{1}-\alpha_{2} & 3^{2}-\alpha_{3} & \alpha_{1}-\alpha_{4} \\
\alpha_{2}-\alpha_{3} & \alpha_{1}-\alpha_{4} & 5^{2}-\alpha_{5}
\end{array}\right]
$$




$$
A^{I I}=\left[\begin{array}{ccc}
2^{2}-\alpha_{2} & \alpha_{1}-\alpha_{3} & \alpha_{2}-\alpha_{4} \\
\alpha_{1}-\alpha_{3} & 4^{2}-\alpha_{4} & \alpha_{1}-\alpha_{5} \\
\alpha_{2}-\alpha_{4} & \alpha_{1}-\alpha_{5} & 6^{2}-\alpha_{6}
\end{array}\right] .
$$

This splitting is a consequence of the symmetry condition (1.3) and implies that the eigenfunctions are either odd or even functions around $\pi / 2$.

It is easy to suggest several algorithms for solving the inverse matrix problem. Our choice is influenced by the convergence proof presented below. Let $\lambda$ be the lowest eigenvalue of $A^{I}$ and partition $A^{I}-\lambda$ as

$$
\left[\begin{array}{cc}
a_{11}-\lambda & a_{1}^{T} \\
a_{1} & A_{1}-\lambda
\end{array}\right]
$$

If $\lambda$ is not an eigenvalue of $A_{1}$, then $\operatorname{det}\left(A^{I}-\lambda\right)=0$ is equivalent to $a_{11}-\lambda=$ $a_{1}^{T}\left(A_{1}-\lambda\right)^{-1} a_{1}$. Since $a_{11}=1-\alpha_{1}$, this is a nonlinear equation which $\alpha_{1}$ must satisfy. Similar arguments for the higher eigenvalues lead to:

The Basic Nonlinear Equations. Let $\lambda_{1}, \lambda_{2}, \ldots, \lambda_{n}$ be the eigenvalues of $A=$ $D+E$, then

$$
\alpha_{\nu}=\nu^{2}-\lambda_{\nu}-a_{\nu}^{T}\left(A_{\nu}-\lambda_{\nu}\right)^{-1} a_{\nu}
$$

for $\nu=1,2, \ldots, m$. If $\nu$ is odd, then $A_{\nu}$ is a submatrix of $A^{I}$ and obtained by deleting the $(\nu+1) / 2$ th row and column of $A^{I}$. The vector $a_{\nu}$ is the $(\nu+1) / 2$ th column of $A^{I}$, except for the diagonal element, which is deleted. If $\nu$ is even, then $A_{\nu}$ is the submatrix of $A^{I I}$ obtained by deleting the $\nu / 2$ th row and column of $A^{I I}$, and the vector $a_{\nu}$ is the $\nu / 2$ th column of $A^{I I}$, except for the diagonal element, which is deleted.

We have now reduced the inverse Sturm-Liouville problem for a differential equation to finding a solution of a system of $m$ nonlinear equations $\alpha=F(\alpha)$. We will show that if $\Sigma\left(\lambda_{j}-j^{2}\right)^{2}$ is small then the functional iteration new $\alpha=F$ (old $\alpha$ ) will converge. We can then prove the intricate part namely that the potentials which are defined by the Fourier coefficients $\alpha$ converge toward the solution of the inverse Sturm-Liouville problem as the dimension of the space of test-functions increases.

2. Eigenvalue Estimates. It is well known that the eigenvalues of the differential equation (1.1) with the Dirichlet boundary conditions (1.2) are simple, but this may fail for our Rayleigh-Ritz procedure. In this section we will find that if the $L^{2}$ norm of the potential is less than (3/4) $\sqrt{2 \pi}$ then the eigenvalues in the Rayleigh-Ritz procedure are simple. Moreover, we will show that the eigenvalues of the matrix $A$ have the same asymptotic behavior as the eigenvalues of the differential equation. Roughly speaking, it is this property which ensures the convergence of the solutions of the discrete problems.

LEMmA 1. Let $\lambda_{\nu}$ be the eigenvalues of $A$ and set $\alpha^{T}=\left(\alpha_{1}, \ldots, \alpha_{n}\right)$. Here $\alpha_{m+1}=\ldots=\alpha_{n}=0$ in case $m<n$. If $\gamma=\|\alpha\|_{2} \leqslant 0.5$, then 


$$
\begin{aligned}
& \left|\lambda_{1}-1+\alpha_{1}\right| \leqslant \frac{\gamma^{2}}{1.5}, \quad\left|\lambda_{2}-2^{2}+\alpha_{2}\right| \leqslant \frac{\gamma^{2}}{2.5}, \\
& \left|\lambda_{\nu}-\nu^{2}+\alpha_{\nu}\right| \leqslant \frac{\gamma^{2}}{\nu-1.5}, \quad \nu=3,4, \ldots, n .
\end{aligned}
$$

Remark. A slightly sharper result can be obtained by using the Temple-Kato brackets, see [17]. Borg has shown that the eigenvalues of the differential equation (1.1) satisfy $\lambda_{\nu}=\nu^{2}-\alpha_{\nu}+o(1 / \nu)$, see [4, p. 11]. Thus the eigenvalues of the two problems have the same asymptotic behavior.

Proof. Let $A=D+E$ where $D=\operatorname{diag}\left(1,2^{2}, \ldots\right)$ and let $e_{\nu}$ be the $\nu$ th column of the identity matrix $I$. Since $A$ is symmetric we find that each disk $\left|\lambda-\nu^{2}\right| \leqslant\left\|E e_{\nu}\right\|_{2}$ contains an eigenvalue. Here $\|x\|_{2}^{2}=\Sigma x_{j}^{2}$. It follows from the definition (1.6) of $A$ that the Fourier coefficient $\alpha_{k}$ can occur at most twice in each column of $A$ and consequently $\left\|E e_{\nu}\right\|_{2} \leqslant 2 \gamma$. We can therefore conclude that if $\gamma<3 / 4$, then the eigenvalues of $A$ are simple and satisfy

$$
\left|\lambda_{\nu}-\nu^{2}\right| \leqslant 2 \gamma
$$

for all $\nu$. Since $A_{\nu}$ has the same structure as $A$, we find that if $j \neq \nu$, then each disk $\left|\lambda-j^{2}\right| \leqslant 2 \gamma$ contains exactly one eigenvalue of $A_{\nu}$. By using these estimates and $\gamma \leqslant 1 / 2$, we obtain

$$
\left\|\left(A_{\nu}-\lambda_{\nu}\right)^{-1}\right\|_{2} \leqslant \frac{1}{4(\nu-1.5)}
$$

for $\nu \geqslant 3$. The bounds for $\nu=1$ and 2 are equal to the bounds for $\nu=3$ and 4 . To complete the proof we use the inequality (2.2) to estimate the last term in Eq. (1.7) and note that $\left\|a_{\nu}\right\|_{2} \leqslant 2 \gamma$.

3. Boundedness of the Successive Approximations. To use the contraction theorem for $\alpha=F(\alpha)$ we must prove two facts. The first is that $F$ maps a sphere into itself and the second is that $F$ is a contraction in the sphere. In this section we will show that if $\Sigma\left(\lambda_{j}-j^{2}\right)^{2}$ is sufficiently small then all successive approximations, new $\alpha=F($ old $\alpha)$, stay within a small sphere whose radius is independent of $n$ and $m$.

Lemma 2. Assume that $\sqrt{\Sigma\left(\lambda_{j}-j^{2}\right)^{2}} \leqslant 0.192$ and consider the functional iteration scheme

$$
\beta_{\nu}=\nu^{2}-\lambda_{\nu}-a_{\nu}^{T}\left(A_{\nu}-\lambda_{\nu}\right)^{-1} a_{\nu}
$$

for $v=1,2, \ldots, m$. Here $m \leqslant n$ and $\beta_{m+1}=\ldots=\beta_{n}=0$ in case $m<n$. If $\|\alpha\|_{2} \leqslant 0.32$, then $\|\beta\|_{2} \leqslant 0.32$.

Proof. Let $\gamma=\|\alpha\|_{2}$ and $\kappa^{2}=\Sigma\left(\lambda_{j}-j^{2}\right)^{2}$. We observe first that the proof of the bound (2.2) uses the fact that $\lambda_{\nu}$ are the eigenvalues of $A$; but it enters only via the inequality (2.1), and this can be replaced by $\left|\lambda_{\nu}-\nu^{2}\right| \leqslant 0.192$. The bound (2.2) is therefore still valid, and by using the arguments from Lemma 1 we get

$$
\Sigma\left(\lambda_{\nu}-\nu^{2}+\beta_{\nu}\right)^{2} \leqslant \gamma^{4}\left[\frac{1}{1.5^{2}}+\frac{1}{2.5^{2}}+\sum_{\nu \geqslant 3} \frac{1}{(\nu-1.5)^{2}}\right] .
$$


The second factor on the right-hand side of (3.2) is less than $25 / 16$, and we conclude by using the triangle inequality that $\|\beta\|_{2} \leqslant \kappa+1.25 \gamma^{2}$. The proof is then completed by inserting the specific values of $\gamma$ and $\kappa$.

In the next section we will show that there exists a solution $\alpha$ of Eq. (1.7) with $\|\alpha\|_{2} \leqslant 0.32$. It then follows from Lemma $1-$ or Eq. (3.2) with $\alpha=\beta-$ that $|\gamma-\kappa| \leqslant(5 / 4) \cdot 0.32 \gamma$ and we obtain the well-posedness result

Corollary 1. Let $\lambda_{j}$ be the eigenvalues of $A$. If $\|\alpha\|_{2} \leqslant 0.32$, then

$$
0.6\|\alpha\|_{2} \leqslant \sqrt{\Sigma\left(\lambda_{j}-j^{2}\right)^{2}} \leqslant 1.4\|\alpha\|_{2} .
$$

This corollary is false without the bound on $\alpha$. For example, if $n=m=3$, then the matrices corresponding to $\alpha=(0,0,0)$ and $\alpha=(-4,0,4)$ have the eigenvalues 1,4 and 9.

4. The Lipschitz Condition. In the previous section we have seen that if $\sqrt{\Sigma\left(\lambda_{j}-j^{2}\right)^{2}} \leqslant 0.192$, then the function $F$ defined by (3.1) maps the sphere $S=$ $\left\{\|\alpha\|_{2} \leqslant 0.32\right\}$ into itself. Since $F$ depends continuously on $\alpha$ we conclude from the Brouwer fixed point theorem that there exists at least one $\alpha$ in $S$ such that $\alpha=F(\alpha)$. We will now prove that $F$ is a contraction in $S$. Thus $F$ has exactly one fixed point in $S$ and it can be obtained by the method of successive approximations.

Let $\alpha$ and $\tilde{\alpha}$ be given, and define $\beta$ and $\widetilde{\beta}$ by

$$
\begin{aligned}
& \beta_{\nu}=\nu^{2}-\lambda_{\nu}-a_{\nu}^{T}\left(A_{\nu}-\lambda_{\nu}\right)^{-1} a_{\nu}, \\
& \tilde{\beta}_{\nu}=\nu^{2}-\lambda_{\nu}-\tilde{a}_{\nu}^{T}\left(\widetilde{A}_{\nu}-\lambda_{\nu}\right)^{-1} \tilde{a}_{\nu} .
\end{aligned}
$$

THEOREM 1. Let $\sqrt{\Sigma\left(\lambda_{j}-j^{2}\right)^{2}} \leqslant 0.192$, and assume that $\|\alpha\|_{2} \leqslant 0.32$ and $\|\widetilde{\alpha}\|_{2} \leqslant 0.32$. If $\beta$ and $\widetilde{\beta}$ are defined by Eqs. (4.1) and (4.2), then

$$
\|\beta-\widetilde{\beta}\|_{2} \leqslant 0.81\|\alpha-\tilde{\alpha}\|_{2} \text {. }
$$

Proof. By subtracting (4.2) from (4.1) we get

$$
\begin{aligned}
\beta_{\nu}-\tilde{\beta}_{\nu}= & \left(\tilde{a}_{\nu}-a_{\nu}\right)^{T}\left(\tilde{A}_{\nu}-\lambda_{\nu}\right)^{-1} \tilde{a}_{\nu}+a_{\nu}^{T}\left(A_{\nu}-\lambda_{\nu}\right)^{-1}\left(\tilde{a}_{\nu}-a_{\nu}\right) \\
& +a_{\nu}^{T}\left(\tilde{A}_{\nu}-\lambda_{\nu}\right)^{-1}\left[A_{\nu}-\tilde{A}_{\nu}\right]\left(A_{\nu}-\lambda_{\nu}\right)^{-1} \tilde{a}_{\nu} .
\end{aligned}
$$

Let $\beta-\widetilde{\beta}=\xi+\eta+\zeta$ where the $\nu$ th terms of $\xi, \eta$ and $\zeta$ are given by the right-hand side of Eq. (4.3). Since the coefficient $\tilde{\alpha}_{k}-\alpha_{k}$ occurs at most twice in the vector $\tilde{a}_{\nu}-a_{\nu}$, we conclude by using Eq. (2.2) that $\left|\xi_{\nu}\right|$ and $\left|\eta_{\nu}\right|$ are less than $0.32\|\tilde{\alpha}-\alpha\|_{2} /(\nu-1.5)$ for $\nu \geqslant 3$. We can now use the arguments from Eq. (3.2) and this leads to

$$
\|\xi\|_{2}+\|\eta\|_{2} \leqslant 0.8\|\alpha-\tilde{\alpha}\|_{2} .
$$

It is more difficult to estimate the last term in Eq. (4.3). The reason is that $\left\|A_{\nu}-\widetilde{A}_{\nu}\right\|_{2}$ cannot be bounded independently of $n$ and $m$. To circumvent this problem we let $D_{\nu}=\operatorname{diag}\left(\ldots,(\nu-2)^{2},(\nu+2)^{2}, \ldots\right)$ and define two real, diagonal matrices $\Delta_{\nu}$ and $I_{\nu}$ by 


$$
\Delta_{\nu}^{2} I_{\nu}=D_{\nu}-\nu^{2} .
$$

Here the diagonal elements of $I_{\nu}$ are either -1 or +1 . By writing $A_{\nu}=D_{\nu}+E_{\nu}$ and dropping the index $\nu$ whenever convenient we get

$$
\begin{gathered}
A_{\nu}-\lambda_{\nu}=\Delta\left(I_{\nu}+\Delta^{-2}\left(\nu^{2}-\lambda_{\nu}\right)+\Delta^{-1} E \Delta^{-1}\right) \Delta, \\
\zeta_{\nu}=a_{\nu}^{T} \Delta^{-1}\left(I_{\nu}+\ldots\right)^{-1} \Delta^{-1}(E-\widetilde{E}) \Delta^{-1}\left(I_{\nu}+\ldots\right)^{-1} \Delta^{-1} \tilde{a}_{\nu} .
\end{gathered}
$$

Let $\|A\|_{F}=\left(\Sigma_{i j} a_{i j}^{2}\right)^{1 / 2}$ be the Frobenius norm of a matrix $A$. Since the Euclidean norm is dominated by the Frobenius norm, we obtain the very useful estimate

$$
\left\|\Delta^{-1} E \Delta^{-1}\right\|_{2} \leqslant\left\|\Delta^{-1}\right\|_{2}\left\|\Delta^{-1}\right\|_{F} \max _{j}\left\|E e_{j}\right\|_{2} .
$$

This result can also be derived by using Gershgorin's theorem. We will now show that the bound (4.7) is in essence independent of $n$ and $m$. From Eq. (4.5) it follows that

$$
\left\|\Delta_{\nu}^{-1}\right\|_{2}^{2}=\frac{1}{4(\nu-1)}
$$

for $\nu \geqslant 3$. For $\nu=1$ and 2 the values are $1 / 8$ and $1 / 12$. To estimate $\left\|\Delta^{-1}\right\|_{F}$ we consider $\Sigma\left|j^{2}-\nu^{2}\right|^{-1}$. Here $j \neq \nu$ and the sum is taken over all positive integers $j$ which have the same parity as $\nu$. By expressing $\left(j^{2}-\nu^{2}\right)^{-1}$ by partial fractions and cancelling as many terms in the sum as possible, we find that

$$
\left\|\Delta_{\nu}^{-1}\right\|_{F}^{2} \leqslant \frac{1}{2 \nu}\left[1+\frac{1}{2}+\ldots+\frac{1}{\nu-1}\right]-\frac{(-1)^{\nu}}{4 \nu^{2}}
$$

for $\nu \geqslant 2$. The bound for $\nu=1$ is $1 / 4$. The largest value of the right-hand side of (4.8) occurs for $\nu=3$ and is $10 / 36$. We also observe that $\left\|\Delta^{-1}\right\|_{2}$ is less than $1 / \sqrt{8}$ for all $\nu$. By combining these estimates with Eq. (4.7) and using that $\alpha_{k}-\tilde{\alpha}_{k}$ can occur at most twice in a given column of $E-\widetilde{E}$, we conclude from Eq. (4.6) that

$$
\left|\zeta_{\nu}\right| \leqslant \frac{0.052}{\nu-1}\|\alpha-\tilde{\alpha}\|_{2}
$$

for $\nu \geqslant 3$. The bounds for $\zeta_{1}$ and $\zeta_{2}$ are equal to the bounds for $\zeta_{3}$ and $\zeta_{4}$. By using (4.9) we find that $\|\zeta\|_{2} \leqslant 0.053\|\alpha-\tilde{\alpha}\|_{2}$. The proof is then completed by combining this result with the estimate (4.4).

At this point, we are halfway through the investigation. Our main result so far is that the inverse matrix problem has a solution. Clearly, it may have many solutions, but the successive approximation method with zero initial guess singles out the solution with the smallest norm. Our method of proof has been inspired by the work of Hadeler [11], Laborde [20], Oliveira [26] and Morel [24]. The main difference occurs in the formulation of the inverse problem. It should also be observed that our estimates are independent of the number of Fourier coefficients and the dimension of the space of trial functions. Finally we mention that Borg solved the inverse Sturm-Liouville problem by a method of successive approximations, see [4, pp. 7181]. His proof is based on the Volterra integral representation of the differential equation (1.1), and our approach can be considered as a numerical interpretation of Borg's idea. 
5. The Fourier Coefficients Converge. Roughly speaking, the solution of the inverse matrix problem is just some numbers $\alpha_{k}$ which are used in Eq. (1.5) to define a potential. The data $\lambda_{j}$ are not the eigenvalues corresponding to this potential. In this section we will show that each Fourier coefficient $\alpha_{k}$ converges as the dimension of the space of trial functions in the Rayleigh-Ritz method increases. It follows from this result that $\lambda_{j}$ are the eigenvalues corresponding to the potential which is defined from the limits of the $\alpha_{k}$.

Lemma 3. Let $\lambda_{1}, \ldots, \lambda_{m}$ be given and consider the solutions $\alpha$ and $\tilde{\alpha}$ of the nonlinear equations

$$
\begin{aligned}
& \alpha_{\nu}=\nu^{2}-\lambda_{\nu}-a_{\nu}^{T}\left(A_{\nu}-\lambda_{\nu}\right)^{-1} a_{\nu}, \\
& \tilde{\alpha}_{\nu}=\nu^{2}-\lambda_{\nu}-\tilde{a}_{\nu}^{T}\left(\widetilde{A}_{\nu}-\lambda_{\nu}\right)^{-1} \tilde{a}_{\nu},
\end{aligned}
$$

for $\nu=1,2, \ldots, m$. Here $\alpha_{k}=\tilde{\alpha}_{k}=0$ for $k \geqslant m$. Let the dimensions of the space of trial functions be $n_{1}$ and $n_{2}$, respectively. Then

$$
\|\alpha-\tilde{\alpha}\|_{2} \leqslant \frac{4}{2+\min \left(n_{1}, n_{2}\right)} \text {. }
$$

Proof. Assume that $n_{1}>n_{2}$. For each $\nu$ we partition the matrix $A_{\nu}-\lambda_{\nu}$ and the vector $a_{\nu}$ as

$$
\left[\begin{array}{cc}
A & B^{T} \\
B & C
\end{array}\right] \text { and }\left[\begin{array}{l}
f \\
g
\end{array}\right] .
$$

Here the partition is chosen such that $A$ and $\widetilde{A}_{\nu}$ have the same dimension. Clearly, $A, B, C, f$ and $g$ depend on $\nu$ but we suppress this dependence to simplify our notation. By solving $\left(A_{\nu}-\lambda_{\nu}\right) x=a_{\nu}$ we can express the last term in Eq. (5.1) as $x^{T} a_{\nu}$ and get

$$
\begin{aligned}
f^{T}\left(A-B^{T} C^{-1} B\right)^{-1} f-2 f^{T}\left(A-B^{T} C^{-1} B\right)^{-1} B^{T} C^{-1} g \\
+g^{T} C^{-1} g+g^{T} C^{-1} B\left(A-B^{T} C^{-1} B\right)^{-1} B^{T} C^{-1} g .
\end{aligned}
$$

If $n_{2}>3 m$, then $g=0$ and the expression simplifies considerably. In practice the solutions become acceptable for much lower values of $n_{2}$, and we will therefore not use this possible shortcut in the proof. It is natural to compare the matrices $A$ and $\widetilde{A}_{\nu}-\lambda_{\nu}$, and by using the above result we find by subtracting Eq. (5.2) from Eq. (5.1) that

$$
\begin{aligned}
\alpha_{\nu}-\tilde{\alpha}_{\nu}= & \tilde{a}_{\nu}^{T}\left(\tilde{A}_{\nu}-\lambda_{\nu}\right)^{-1} \tilde{a}_{\nu}-f^{T} A^{-1} f \\
& -f^{T}\left(A-B^{T} C^{-1} B\right)^{-1} B^{T} C^{-1} B A^{-1} f+2 f^{T}\left(A-B^{T} C^{-1} B\right)^{-1} B^{T} C^{-1} g \\
& -g^{T} C^{-1} g-g^{T} C^{-1} B\left(A-B^{T} C^{-1} B\right)^{-1} B^{T} C^{-1} g
\end{aligned}
$$

Let $\alpha-\tilde{\alpha}=\eta^{(1)}+\ldots+\eta^{(6)}$ where the $\nu$ th term of $\eta^{(1)}, \ldots, \eta^{(6)}$ are given by the right-hand side of Eq. (5.3). The matrix $A$ is equal to the matrix which we would have obtained if $n_{1}$ and $n_{2}$ had been equal. Theorem 1 is therefore applicable and we conclude that 


$$
\left\|\eta^{(1)}+\eta^{(2)}\right\|_{2} \leqslant 0.81\|\alpha-\tilde{\alpha}\|_{2} .
$$

To estimate the last four terms in Eq. (5.3) we modify the technique used in the proof of Theorem 1. Let $\Sigma$ be the diagonal matrix defined by $\Sigma^{2}=\operatorname{diag}\left((n+1)^{2}\right.$ $\left.-\nu^{2},(n+3)^{2}-\nu^{2}, \ldots\right)$. Here $n=n_{2}+1$ if $\nu$ and $n_{2}$ have the same parity and $n=n_{2}$ otherwise. Since $\nu \leqslant m \leqslant n$, we can choose $\Sigma$ such that its diagonal elements are positive. We will now factorize the matrices $A$ and $C$ in the form

$$
\begin{aligned}
& A=\Delta\left(I_{\nu}+\Delta^{-2}\left(\nu^{2}-\lambda_{\nu}\right)+\Delta^{-1} E \Delta^{-1}\right) \Delta, \\
& C=\Sigma\left(I+\Sigma^{-2}\left(\nu^{2}-\lambda_{\nu}\right)+\Sigma^{-1} F \Sigma^{-1}\right) \Sigma .
\end{aligned}
$$

Here $\Delta$ and $I_{\nu}$ are defined by Eq. (4.5). By letting $W=\Sigma^{-1} B \Delta^{-1}$ we can rewrite the third term on the right-hand side of Eq. (5.3) as

$$
-f^{T} \Delta^{-1}\left[I_{\nu}+R-W^{T}(I+S)^{-1} W\right]^{-1} W^{T}(I+S)^{-1} W\left(I_{\nu}+R\right)^{-1} \Delta^{-1} f .
$$

Here $R=\Delta^{-2}\left(\nu-\lambda_{\nu}\right)+\Delta^{-1} E \Delta^{-1}$ and $S$ is defined similarly. Since $\left\|\Delta^{-1}\right\|_{2} \leqslant 1 / \sqrt{8}$ and $\left\|\Delta^{-1}\right\|_{F} \leqslant \sqrt{10} / 6$ for all $\nu$, we conclude from Eq. (4.7) that $\|R\|_{2} \leqslant 0.024+$ $\sqrt{5} \cdot 0.16 / 3$. This bound is also valid for $\|S\|_{2}$, simply because $\Sigma$ is a submatrix of a very large $\Delta$. To estimate the norm of $W$ we use that the Frobenius norm dominates the Euclidean norm and obtain

$$
\|W\|_{2} \leqslant\left\|\Sigma^{-1}\right\|_{2}\left\|\Delta^{-1}\right\|_{F} \max _{j}\left\|B e_{j}\right\|_{2} .
$$

This generalizes Eq. (4.7). It follows from the definition of $\Sigma$ that $\left\|\Sigma^{-1}\right\|_{2}^{2} \leqslant$ $1 /\left[(n+1)^{2}-\nu^{2}\right]$ where $n_{c}=n_{2}$. We remember now that any Fourier coefficient $\alpha_{k}$ can occur at most twice in a column of $B$ and, hence $\left\|B e_{j}\right\|_{2} \leqslant 2 \cdot 0.32$. By using Eq. (5.6) and the bounds for $R, S, \Delta$ and $\Sigma$ we find from (5.5) that the third term on the right-hand side of Eq. (5.3) can be estimated by

$$
\left|\eta_{\nu}^{(3)}\right| \leqslant \frac{0.0095}{(n+1)^{2}-\nu^{2}} .
$$

The fourth, fifth and sixth terms on the right-hand side of Eq. (5.3) can be estimated in the same manner, and we state without further ado that

$$
\left|\eta_{\nu}^{(4)}\right| \leqslant \frac{0.068}{(n+1)^{2}-\nu^{2}}, \quad\left|\eta_{\nu}^{(5)}\right| \leqslant \frac{0.48}{(n+1)^{2}-\nu^{2}}, \quad\left|\eta_{\nu}^{(6)}\right| \leqslant \frac{0.0095}{(n+1)^{2}-\nu^{2}} .
$$

Since $1 \leqslant \nu \leqslant m \leqslant n$ and $(n+1)^{2}-\nu^{2} \geqslant(n+2)(n+1-\nu)$, we see that

$$
\sum_{\nu=1}^{m} \frac{1}{\left[(n+1)^{2}-\nu^{2}\right]^{2}} \leqslant \frac{\pi^{2}}{6} \frac{1}{n+2} \text {. }
$$

By combining this result with the estimates of the $\nu$ th component of $\eta^{(3)}, \ldots, \eta^{(6)}$ we conclude that

$$
\left\|\eta^{(3)}+\ldots+\eta^{(6)}\right\|_{2} \leqslant \frac{0.73}{n+2}
$$


Finally, it follows from Eq. (5.3) and Eq. (5.4) that $0.19\|\alpha-\tilde{\alpha}\|_{2}$ is less than the bound given in Eq. (5.7). This completes the proof.

Lemma 3 shows that the sequence of vectors $\alpha$ which depend on the dimension $n$ of the space of trial functions is a Cauchy sequence in $R^{m}$ and hence converges. Let $q(x)$ be the potential corresponding to the limits of the Fourier coefficients, and let $q_{n}$ be the potential determined by (1.5). By using Lemma 3 and Parseval's theorem we see that

$$
\left\|q-q_{n}\right\|_{2} \leqslant \frac{10}{n+2}
$$

This result is true for all $n \geqslant m$. However, it follows from the proof of Lemma 3 that if $n>3 m$, then the bound in Eq. (5.8) can be replaced by $0.16 /(n+2)$. The reason is that the vector $g$ in Eq. (5.3) vanishes. Finally, numerical experiments indicate that both estimates are quite generous, but this is to be expected.

6. Proof of Ellipticity. In the previous section we have seen that the potentials, which correspond to the computed Fourier coefficients, converge in $L^{2}$ to a potential $q(x)$, as we increase the dimension of the space of trial functions. In the next section we will show that $\lambda_{j}$ will be the eigenvalues corresponding to this limit potential. The proof will be based on some simple upper and lower bounds for the quadratic form $a(u, u)=\int_{0}^{\pi} u^{\prime 2}+q u^{2}$. The topic of this section is to establish those bounds.

Lemma 4. Assume that $q(x)=q(\pi-x)$ for almost all $x$. If $u(0)=u(\pi)=0$ and $\|q\|_{2} \leqslant 0.32 \sqrt{2 \pi}$, then

$$
0.28 \int_{0}^{\pi} u^{\prime 2} \leqslant a(u, u) \leqslant 1.72 \int_{0}^{\pi} u^{\prime 2} .
$$

Remark. This result shows that $\sqrt{a(u, u)}$ is a norm on the space of smooth functions which vanishes at 0 and $\pi$ and equivalent to $\left(\int_{0}^{\pi} u^{\prime 2}\right)^{1 / 2}$. We denote the completion of this space by $H^{1}$. Note that we do not assume that $q$ is a bounded function, cf. Ciarlet, Schultz and Varga [5].

Proof. Let $u$ and $v$ be smooth functions and vanish at 0 and $\pi$. It follows from the Cauchy-Schwarz inequality that

$$
\left|\int_{0}^{\pi} q u v\right|^{2} \leqslant \int_{0}^{\pi}|q|^{2}|u|^{2} \int_{0}^{\pi}|v|^{2} .
$$

The last factor on the right-hand side of (6.1) can be estimated by $\int_{0}^{\pi}\left|v^{\prime}\right|^{2}$, see [14, p. 185]. To estimate the first factor we note that $|u(x)|^{2} \leqslant(\pi / 2) \int_{0}^{\pi / 2}\left|u^{\prime}\right|^{2}$ for all $x$ in $[0, \pi / 2]$. A similar Sobolev inequality holds for the interval $[\pi / 2, \pi]$. By combining these results and using the symmetry of $q(x)$ we conclude from Eq. (6.1) that

$$
\left|\int_{0}^{\pi} q u v\right|^{2} \leqslant \frac{\pi}{4} \int_{0}^{\pi}|q|^{2} \int_{0}^{\pi}\left|u^{\prime}\right|^{2} \int_{0}^{\pi}\left|v^{\prime}\right|^{2} .
$$

We can now establish the upper bound. Let $u=v$. Since $\|q\|_{2} \leqslant 0.32 \sqrt{2 \pi}$, we find from Eq. (6.2) that 


$$
a(u, u)=\int_{0}^{\pi} u^{\prime 2}+q u^{2} \leqslant\left(1+\frac{\sqrt{\pi}}{2} \cdot 0.32 \sqrt{2 \pi}\right)\left\|u^{\prime}\right\|_{2}^{2} .
$$

The lower bound is obtained in a similar manner, and this completes the proof.

Lemma 4 shows that the operator $-u^{\prime \prime}+q u$ is elliptic and that the lowest eigenvalue $\mu_{1}$ is greater than 0.28 . More precisely, it follows from Corollary 1 that $\mu_{1} \geqslant 0.55$. Both results have been derived by using the symmetry assumption for the potential, and it is not clear whether Lemma 4 is valid without this assumption.

Let $\tilde{q}$ be a symmetric potential and define the quadratic form $\tilde{a}$ by $\tilde{a}(u, u)=$ $\int_{0}^{\pi} u^{\prime 2}+\widetilde{q u}{ }^{2}$. We will now show that if $q-\tilde{q}$ is small, then the ratio $a / \tilde{a}$ of the quadratic form is close to one.

Lemma 5. Let $u$ be in $H^{1}$ and satisfy the boundary conditions $u(0)=u(\pi)=$ 0 . Assume that $q(x)=q(\pi-x)$ and $\tilde{q}(x)=\tilde{q}(\pi-x)$ for almost all $x$ in $[0, \pi]$. If $\|q\|_{2}$ and $\|\tilde{q}\|_{2}$ are less than $0.32 \sqrt{2 \pi}$, then

$$
\left|\frac{a(u, u)}{\tilde{a}(u, u)}-1\right| \leqslant 3.2\|q-\tilde{q}\|_{2} .
$$

Proof. It follows from the definition of $a$ and $\tilde{a}$ that

$$
\tilde{a}(u, u)=a(u, u)+\int_{0}^{\pi}(\tilde{q}-q) u^{2} .
$$

According to (6.2), we can estimate the last term in (6.3) by $(\sqrt{\pi} / 2)\|q-\widetilde{q}\|_{2}\left\|u^{\prime}\right\|_{2}^{2}$. The proof is then completed by using the lower bound in Lemma 4 .

7. Solution of the Inverse Sturm-Liouville Problem. We have seen that given $\lambda_{1}, \ldots, \lambda_{m}$ the inverse matrix problem has a solution for each space of trial functions with dimension greater than or equal to $m$. The corresponding potentials have been constructed such that $\lambda_{j}$ are the eigenvalues obtained by the Rayleigh-Ritz method. However, in general $\lambda_{j}$ will not be the eigenvalues of the differential equation (1.1) with boundary conditions (1.2). Instead, we have shown that the potentials converge to a limit potential as we increase the dimension of the space of trial functions. In this section we will prove the main result of this paper, namely that $\lambda_{j}$ are the eigenvalues corresponding to the limit potential. In practice we will never find this potential, but according to Eq. (5.8) we can approximate it arbitrarily well. It is in this sense that we solve the inverse Sturm-Liouville problem.

THEOREM 2. Let $\sqrt{\Sigma\left(\lambda_{j}-j^{2}\right)^{2}} \leqslant 0.192$ and let $q_{n}(x)$ be the potential (1.5) defined from the solution of the inverse matrix problem. Here $n$ is the dimension of the space of trial functions. Let $q(x)=\lim q_{n}(x)$. Then $\lambda_{j}$ are the eigenvalues of the differential equation (1.1) with boundary conditions (1.2).

Proof. The proof is based on the technique developed for the finite element method, in particular see Strang and Fix [27, Chapter 6]. Let $\mu_{j}$ be the eigenvalues of the differential equation (1.1) with the potential $q(x)$. We will show that $\mu_{j}=\lambda_{j}$. Let $S_{l}$ be an $l$-dimensional subspace of $H^{1}$, where $H^{1}$ is defined in the remark following Lemma 4. According to the minimax principle [27, p. 221], we can characterize the $l$ th eigenvalue by 


$$
\mu_{l}=\min _{s_{l}} \max _{u \in s_{l}} \frac{a(u, u)}{(u, u)} .
$$

Let $q_{n}=\tilde{q}$ and let $\tilde{a}$ be the corresponding quadratic form. By interchanging the role of $a$ and $\tilde{a}$ in Lemma 4 and using Eq. (7.1) we find

$$
\mu_{l} \leqslant\left(1+3.2\|q-\tilde{q}\|_{2}\right) \min _{s_{l}} \max _{u \in s_{l}} \frac{\tilde{a}(u, u)}{(u, u)} .
$$

Let $S$ be the space of functions $u$ of the form $\Sigma_{1}^{n} w_{j} \sin j x$. If $u$ is in $S$, then $\tilde{a}(u, u)$ $=w^{T} A w$ where $A$ is defined as in Section 1. We choose now $S_{l}$ to be the subspace of $S$ which is spanned by the first $l$ eigenvectors of $A$. The potential $q_{n}$ has been constructed such that $\lambda_{j}$ are the eigenvalues of $A$. We can therefore conclude from the minimax principle and Eq. (7.2) that $\mu_{l} \leqslant\left(1+3.2\left\|q-q_{n}\right\|_{2}\right) \lambda_{l}$. Since $q_{n} \rightarrow q$, this inequality shows that $\mu_{l} \leqslant \lambda_{l}$ for $l=1,2, \ldots, m$.

It is more difficult to prove the opposite inequality. Let $u$ be in $H^{1}$ and define the projection $P u$ of $u$ onto $S$ by

$$
a(u-P u, v)=0
$$

for all $v$ in $S$. It can be shown by a standard variational argument that $\|u-P u\|_{2} \rightarrow$ 0 as the dimension of $S$ increases, see [27, p. 166 and p. 229]. The argument is known as Nitsche's trick, and the proof is based on expanding the function $u$ in a Fourier sine series and uses Lemma 4.

Let $E_{l}$ be the space spanned by the first $l$ eigenfunctions $y_{1}, \ldots, y_{l}$ of the differential equation (1.1) with the potential $q(x)$. We assume that all $y_{j}$ are normalized and denote the unit sphere of $E_{l}$ by $E_{l}$. Let

$$
\sigma_{l}=\max _{E_{l}}|2(u, u-P u)-(u-P u, u-P u)| .
$$

Since each $u$ in $E_{l}$ can be expressed in the form $\Sigma_{1}^{l} c_{j} y_{j}$ with $\Sigma c_{j}^{2}=1$ and $\left\|y_{j}-P y_{j}\right\|_{2}$ $\rightarrow 0$ as $n \rightarrow \infty$, we conclude that $\sigma_{l} \rightarrow 0$ as $n \rightarrow \infty$. Moreover, it can be shown that the dimension of $P E_{l}$ is $l$ if $\sigma_{l}<1$, see [27, p. 229]. We can now characterize the $l$ th eigenvalue of the matrix $A$ by the minimax principle and find by using Lemma 5 that

$$
\lambda_{l} \leqslant\left(1+3.2\|q-\tilde{q}\|_{2}\right) \min _{S_{l}} \max _{u \in S_{l}} \frac{a(u, u)}{(u, u)} .
$$

The minimum is taken over all $l$-dimensional subspaces of $S$. Let now $S_{l}$ be the space $P E_{l}$. It follows from Lemma 4 and the definition (7.3) of $P$ that $a(P u, P u) \leqslant$ $a(u, u)$ for all $u$. Since $(P u, P u) \geqslant 1-\sigma_{l}$ for all $u$ in $E_{l}$, we infer from Eq. (7.4) that

$$
\lambda_{l} \leqslant\left(1+3.2\left\|q-q_{n}\right\|\right) \max _{E_{l}} \frac{a(u, u)}{1-\sigma_{l}} .
$$

Since $a(u, u)=\Sigma_{1}^{l} c_{j}^{2} \mu_{j}$ with $\Sigma c_{j}^{2}=1$ we see that the last factor on the right-hand side of (7.5) is less than $\mu_{l} /\left(1-\sigma_{l}\right)$. Finally, by letting $n \rightarrow \infty$ and using that $\sigma_{l} \rightarrow 0$ and $\left\|q-q_{n}\right\|_{2} \rightarrow 0$ we conclude that $\lambda_{l} \leqslant \mu_{l}$. This completes the proof. 
8. Infinitely Many Eigenvalues Prescribed. So far our approach has been consistent with the manner in which inverse problems occur in practice. For example, in geophysics only a finite number of the eigenfrequencies of the earth are measured and used in the inversion procedure. To obtain unique results the number of parameters must be less than or equal to the number of data. In our formulation the uniqueness of the potential is obtained by representing it as a trigonometric polynomial. Clearly, a different representation yields a different potential.

In the classical formulation of the inverse Sturm-Liouville problem it is assumed that all the eigenvalues corresponding to a given potential are known and then slightly perturbed. Borg has shown that there exists a unique nearby potential which corresponds to the perturbed spectrum. Assume, therefore, that all the eigenvalues are given, but that only the first $m$ have been used in our construction. In this section we will show that the effect of the remaining eigenvalues is small. This statement must be taken with a grain of salt, because the whole theory is a perturbation theory. Indeed, there are examples, with large perturbations of the eigenvalues, for which the distance between two potentials cannot be estimated in terms of the difference between the spectra, see Hald [13].

Lemma 6. Assume that $\sqrt{\Sigma\left(\lambda_{j}-j^{2}\right)^{2}} \leqslant 0.192$. Let $\alpha$ be the solution of the inverse matrix problem with data $\lambda_{1}, \ldots, \lambda_{m_{1}}$ and let $\tilde{\alpha}$ correspond to $\lambda_{1}, \ldots, \lambda_{m_{2}}$ with $1<m_{1}<m_{2}$. If the spaces of trial functions have the same dimension, then

$$
\|\alpha-\tilde{\alpha}\|_{2} \leqslant 5.3 \sqrt{\sum_{m_{1}+1}^{m_{2}}\left(\lambda_{j}-j^{2}\right)^{2}}+0.54 \sqrt{\frac{m_{2}-m_{1}}{\left(m_{2}-1.5\right)\left(m_{1}-1.5\right)}} .
$$

Proof. We will use the technique developed in Section 4. Since $\alpha$ and $\tilde{\alpha}$ are solutions of the inverse matrix problem we can replace $\beta_{\nu}$ and $\widetilde{\beta}_{\nu}$ in Eqs. (4.1) and (4.2) with $\alpha_{\nu}$ and $\tilde{\alpha}_{\nu}$. It follows from the proof of Theorem 1 and Eq. (4.3) in particular that

$$
\sqrt{\sum_{1}^{m_{1}}\left(\alpha_{\nu}-\tilde{\alpha}_{\nu}\right)^{2}} \leqslant 0.81\|\alpha-\tilde{\alpha}\|_{2}
$$

Since the $\alpha_{\nu}$ are set equal to zero for $\nu>m_{1}$, we must estimate $\tilde{\alpha}_{\nu}$ from Eq. (4.2), directly. By using (2.2) and the triangle inequality we find that

$$
\sqrt{\sum_{m_{1}+1}^{m_{2}}\left(\tilde{\alpha}_{\nu}\right)^{2}} \leqslant \sqrt{\sum_{m_{1}+1}^{m_{2}}\left(\lambda_{\nu}-\nu^{2}\right)^{2}}+0.32^{2} \sqrt{\sum_{m_{1}+1}^{m_{2}} \frac{1}{(\nu-1.5)^{2}}} .
$$

The proof is now completed by estimating the last sum by an integral and combining Eqs. (8.1) and (8.2).

The above proof did not depend explicitly on the dimension $n$ of the space of trial functions although the Fourier coefficients $\alpha_{\nu}$ and $\tilde{\alpha}_{\nu}$ do. It follows from Lemma 3 that the components of $\alpha$ and $\tilde{\alpha}$ converge as $n \rightarrow \infty$. We can therefore conclude 
from Lemma 6 that the potentials $Q_{m_{1}}(x)$ and $Q_{m_{2}}(x)$ associated with the limit of $\alpha$ and $\tilde{\alpha}$ form a Cauchy sequence in $L^{2}$. By using Parseval's theorem and letting $m_{2}$ tend to $\infty$, we obtain from Lemma 6 the following result:

Corollary 2. Assume that $\sqrt{\Sigma\left(\lambda_{j}-j^{2}\right)^{2}} \leqslant 0.192$. Let $Q_{m}$ be the potential which has the eigenvalues $\lambda_{1}, \ldots, \lambda_{m}$ and let $Q$ be the potential corresponding to $\lambda_{1}, \lambda_{2}, \ldots$ Then

$$
\left\|Q-Q_{m}\right\|_{2} \leqslant 13.3 \sqrt{\sum_{m+1}^{\infty}\left(\lambda_{j}-j^{2}\right)^{2}}+\frac{1.4}{\sqrt{m-1.5}} .
$$

One might object that we have not shown that the limit $Q$ of the potentials $Q_{m}$ has the eigenvalues $\lambda_{1}, \lambda_{2}, \ldots$ However, according to Eq. (5.8) each potential $Q_{m}$ can be approximated arbitrarily well by a potential which corresponds to a solution of an inverse matrix problem. Thus, the proof of Theorem 2 carries over to the present case.

9. Continuous Dependence on the Data. In the previous section we have incidentally obtained an existence theorem for the inverse Sturm-Liouville problem in its classical formulation. Since we perturb around the spectrum corresponding to the zero potential, our result is only local. The proof is independent of the theory for the differential equations. The existence proofs due to Borg [4] and Barcilon [3] are also based on the method of successive approximations, and it is normally easy to derive well-posedness results for these methods. In our case we have

THEOREM 3. Assume that $\sqrt{\Sigma\left(\lambda_{j}-j^{2}\right)^{2}}$ and $\sqrt{\Sigma\left(\widetilde{\lambda_{j}}-j^{2}\right)^{2}}$ are less than 0.192. If $q(x)$ and $\tilde{q}(x)$ are the potentials corresponding to $\lambda_{j}$ and $\tilde{\lambda}_{j}$, then

$$
\|q-\widetilde{q}\|_{2} \leqslant 13.3 \sqrt{\Sigma\left(\lambda_{j}-\widetilde{\lambda}_{j}\right)^{2}}
$$

Remark. This result is valid for the solutions of the inverse Sturm-Liouville problem and the potentials obtained from the inverse matrix problem. Here we assume that the potentials are represented by the same number of Fourier coefficients and that these are computed by using the same space of trial functions.

Proof. Let $\alpha$ and $\tilde{\alpha}$ be the solutions of the inverse matrix problems with data $\lambda_{1}, \ldots, \lambda_{m}$ and $\tilde{\lambda}_{1}, \ldots, \tilde{\lambda}_{m}$. Thus, $\alpha_{\nu}$ satisfy Eq. (4.1) with $\beta_{\nu}$ replaced by $\alpha_{\nu}$ and $\widetilde{\alpha}_{\nu}$ satisfy Eq. (4.2) with $\widetilde{\beta}_{\nu}$ and $\lambda_{\nu}$ replaced by $\widetilde{\alpha}_{\nu}$ and $\widetilde{\lambda}_{\nu}$. By subtracting Eq. (4.2) from Eq. (4.1) we obtain Eq. (4.3) with two additional terms, namely $\lambda_{\nu}-\tilde{\lambda}_{\nu}$ and

$$
a_{\nu}^{T}\left(\tilde{A}-\tilde{\lambda}_{\nu}\right)^{-1}\left(\lambda_{\nu}-\tilde{\lambda}_{\nu}\right)\left(A-\lambda_{\nu}\right)^{-1} \tilde{a}_{\nu}
$$

It follows from Eq. (2.2) that the sum of these two terms must be less than $1.0064\left|\lambda_{\nu}-\tilde{\lambda}_{\nu}\right|$. By using the triangle inequality, Lemma 4 and Parseval's theorem we obtain (9.1).

Let $m$ be fixed. Since the Fourier coefficients $\alpha$ and $\tilde{\alpha}$ converge as $n \rightarrow \infty$, see (5.8), we conclude that the inequality (9.1) holds for the limit potentials. Finally, 
by letting $m \rightarrow \infty$ it follows from Corollary 2 that (9.1) is also valid for the inverse Sturm-Liouville problem in its classical formulation. This completes the proof.

10. Numerical Experiments. In the derivation of our algorithm for the inverse Sturm-Liouville problem we have assumed that the potential is a square integrable function. The theory does not distinguish between smooth and not so smooth potentials, but the difference is very obvious in practice.

Borg has shown, see $[4$, p. 11], that if the potential is twice differentiable then $\lambda_{\nu}=\nu^{2}+$ const $\cdot \nu^{-2}+O\left(\nu^{-3}\right)$, otherwise the $\nu$ th eigenvalue has the asymptotic expansion $\lambda_{\nu}=\nu^{2}-\alpha_{\nu}+o\left(\nu^{-1}\right)$, where $\alpha_{\nu}$ is the $2 \nu$ th Fourier coefficient of the potential. Roughly speaking the consequence of these asymptotic expansions is that a small number of eigenvalues are sufficient to determine the potential if it is smooth, whereas a considerable number is needed if the potential has any kind of discontinuities.

In our theory we have assumed that the average of the potential has been determined from the asymptotic expansion of the eigenvalues. However, if only a finite number of data is available, an ad hoc guess is necessary. This guess may be based on the behavior of the eigenvalues or on some a priori information about the potential. We have not been able to find a solution of this very complicated problem.

The algorithm presented in Section 1 has been tested on three different potentials. In all three cases, the eigenvalues used were correct to eight decimal places. The first test problem is the Mathieu equation with the potential

$$
q_{1}(x)=2 \cos (2 x)
$$

The $L^{2}$ norm of this potential is $\sqrt{2 \pi}$, and therefore this case is not covered by our theory. The potential is atypical because it can be described by a finite Fourier series. On the other hand, the Mathieu equation is important by itself, and the eigenvalues have been carefully tabulated, see [28].

To investigate the importance of the representation of the eigenfunctions we tried to calculate the potential from the first six eigenvalues. The errors in the Fourier coefficients $\alpha_{k}$ can be found in Table 1. We see that the lowest Fourier coefficients are best determined and that it is preferable to choose the dimension of the space of trial functions as an even number.

In Theorem 3 we have shown that the inverse Sturm-Liouville problem is wellposed. However, from a practical point of view the problem must be considered as ill-posed. For example, for the inverse problem for the earth the higher eigenfrequencies are measured with less absolute accuracy than the lower ones, and finally regarded as noise. To investigate this phenomenon we have perturbed the first, fifth and tenth eigenvalue of the Mathieu equation by one percent. The results are given in Table 2. We conclude that $\alpha_{\nu}$ is roughly equal to $\nu^{2}-\lambda_{\nu}$ but that the perturbation spreads out to the nearby Fourier coefficients. In the case where all eigenvalues are perturbed by one percent the solution is completely dominated by noise, yet the lowest Fourier coefficients are essentially correct. 


\section{Dimension of the space of trial functions}

\begin{tabular}{|c|c|c|c|c|c|}
\hline Errors in & 6 & 7 & 8 & 9 & 10 \\
\hline$\alpha_{1}$ & $3.1 \cdot 10^{-6}$ & $6.1 \cdot 10^{-8}$ & $3.3 \cdot 10^{-9}$ & $2.5 \cdot 10^{-9}$ & $2.5 \cdot 10^{-9}$ \\
\hline$\alpha_{2}$ & $4.1 \cdot 10^{-5}$ & $1.1 \cdot 10^{-6}$ & $1.5 \cdot 10^{-8}$ & $3.6 \cdot 10^{-9}$ & $3.4 \cdot 10^{-9}$ \\
\hline$\alpha_{3}$ & $4.3 \cdot 10^{-4}$ & $1.7 \cdot 10^{-5}$ & $2.0 \cdot 10^{-7}$ & $5.5 \cdot 10^{-9}$ & $1.3 \cdot 10^{-9}$ \\
\hline$\alpha_{4}$ & $4.0 \cdot 10^{-3}$ & $2.0 \cdot 10^{-4}$ & $3.1 \cdot 10^{-6}$ & $9.0 \cdot 10^{-8}$ & $1.9 \cdot 10^{-9}$ \\
\hline$\alpha_{5}$ & $4.1 \cdot 10^{-2}$ & $2.9 \cdot 10^{-3}$ & $3.2 \cdot 10^{-6}$ & $1.6 \cdot 10^{-6}$ & $8.0 \cdot 10^{-5}$ \\
\hline$\alpha_{6}$ & $3.1 \cdot 10^{-2}$ & $3.0 \cdot 10^{-2}$ & $1.7 \cdot 10^{-5}$ & $2.0 \cdot 10^{-5}$ & $2.5 \cdot 10^{-6}$ \\
\hline
\end{tabular}

TABLE 1

Errors in the Fourier coefficients

\begin{tabular}{|c|c|c|c|c|c|}
\hline Errors in: & $\begin{array}{l}\text { All } \lambda_{j} \\
\text { exact }\end{array}$ & $\begin{array}{c}\lambda_{1} \\
\text { pert. }\end{array}$ & $\begin{array}{r}\lambda_{5} \\
\text { pert. }\end{array}$ & $\begin{array}{l}\lambda_{10} \\
\text { pert. }\end{array}$ & $\begin{array}{l}\text { All } \lambda_{j} \\
\text { perturbed }\end{array}$ \\
\hline$\alpha_{1}$ & $2.5 \cdot 10^{-9}$ & $8.9 \cdot 10^{-4}$ & $-1.3 \cdot 10^{-3}$ & $-4.0 \cdot 10^{-6}$ & $1.1 \cdot 10^{-2}$ \\
\hline$\alpha_{2}$ & $3.5 \cdot 10^{-9}$ & $-1.3 \cdot 10^{-4}$ & $-1.7 \cdot 10^{-3}$ & $-4.9 \cdot 10^{-5}$ & $1.9 \cdot 10^{-2}$ \\
\hline$\alpha_{3}$ & $1.3 \cdot 10^{-9}$ & $1.1 \cdot 10^{-4}$ & $-3.5 \cdot 10^{-3}$ & $-5.6 \cdot 10^{-4}$ & $-9.8 \cdot 10^{-2}$ \\
\hline$\alpha_{4}$ & $1.4 \cdot 10^{-9}$ & $4.5 \cdot 10^{-5}$ & $-2.8 \cdot 10^{-2}$ & $-4.7 \cdot 10^{-3}$ & $1.4 \cdot 10^{-1}$ \\
\hline$\alpha_{5}$ & $2.3 \cdot 10^{-9}$ & $3.2 \cdot 10^{-5}$ & $-2.5 \cdot 10^{-1}$ & $-5.0 \cdot 10^{-3}$ & $-2.7 \cdot 10^{-1}$ \\
\hline$\alpha_{\varepsilon}$ & $0.2 \cdot 10^{-9}$ & $2.3 \cdot 10^{-5}$ & $2.8 \cdot 10^{-2}$ & $-6.0 \cdot 10^{-3}$ & $3.3 \cdot 10^{-1}$ \\
\hline$\alpha_{7}$ & $-0.6 \cdot 10^{-9}$ & $1.7 \cdot 10^{-5}$ & $2.1 \cdot 10^{-4}$ & $-8.6 \cdot 10^{-3}$ & $-5.0 \cdot 10^{-1}$ \\
\hline$\alpha_{8}$ & $-0.9 \cdot 10^{-9}$ & $1.3 \cdot 10^{-5}$ & $9.5 \cdot 10^{-4}$ & $-1.2 \cdot 10^{-2}$ & $6.1 \cdot 10^{-1}$ \\
\hline$\alpha_{9}$ & $-3.6 \cdot 10^{-9}$ & $1.1 \cdot 10^{-5}$ & $5.6 \cdot 10^{-4}$ & $2.6 \cdot 10^{-2}$ & $-8.0 \cdot 10^{-1}$ \\
\hline$\alpha_{10}$ & $-4.7 \cdot 10^{-9}$ & $8.4 \cdot 10^{-6}$ & $-2.6 \cdot 10^{-5}$ & 1.0 & 1.1 \\
\hline
\end{tabular}

TABLE 2

$$
\text { Perturbation of } \lambda_{j} \text { to } \lambda_{j}-0.01 \cdot \lambda_{j}(-1)^{j}
$$

The second potential was chosen as the Hermite interpolation of $q_{1}(x)$ and then normalized to have zero mean. Thus

$$
q_{2}(x)=\frac{32}{15}-\frac{64}{\pi^{4}} x^{2}(\pi-x)^{2}=\frac{192}{\pi^{4}} \sum_{k=1}^{\infty} \frac{1}{k^{4}} \cos (2 k x) .
$$

The eigenvalues for this potential were computed by the Rayleigh-Ritz method with 60 sine functions spanning the space of trial functions, and the results were judged to be accurate to $10^{-8}$. In Table 3 we give the $l^{2}$ error of the computed Fourier coefficients. One piece of information which cannot be inferred from Table 3 is that the first Fourier coefficients are best determined. Indeed, for the case of 16 eigenvalues 
and 24 basis functions all but the last two of the Fourier coefficients are accurate to within $10^{-8}$. This is interesting because the first Fourier coefficient of the part of the function which we do not take into account is of order $10^{-5}$. One possible reason for this is that the higher eigenvalues are well described by Borg's asymptotic expansion of the eigenvalues corresponding to a smooth potential, and that the term const $\cdot \nu^{-2}$ dominates the Fourier coefficient $\alpha_{\nu}$.

$$
\text { Number of eigenvalues }
$$

\begin{tabular}{|c|c|c|c|c|}
\hline $\begin{array}{l}\text { No. of basis } \\
\text { functions }\end{array}$ & 4 & 8 & 12 & 16 \\
\hline 4 & $7.2 \cdot 10^{-2}$ & & & \\
\hline 8 & $1.5 \cdot 10^{-4}$ & $3.9 \cdot 10^{-2}$ & & \\
\hline 12 & $1.6 \cdot 10^{-4}$ & $7.4 \cdot 10^{-6}$ & $2.7 \cdot 10^{-2}$ & \\
\hline 16 & $1.6 \cdot 10^{-4}$ & $8.2 \cdot 10^{-6}$ & $9.1 \cdot 10^{-7}$ & $2.1 \cdot 10^{-2}$ \\
\hline 20 & & & $1.3 \cdot 10^{-6}$ & $6.4 \cdot 10^{-7}$ \\
\hline 24 & & & $1.3 \cdot 10^{-6}$ & $3.4 \cdot 10^{-7}$ \\
\hline
\end{tabular}

TABLE 3

Errors in the Fourier coefficients: $\sqrt{\Sigma\left(\operatorname{comp} \alpha_{k}-\operatorname{exact} \alpha_{k}\right)^{2}}$

Finally, our third potential was chosen as a piecewise constant approximation of $q_{1}(x)$, specifically

$$
\begin{aligned}
q_{3}(x) & = \begin{cases}-2 & \text { if } \pi / 4<x<3 \pi / 4 \\
2 & \text { otherwise, }\end{cases} \\
& =\frac{8}{\pi} \sum_{k=1}^{\infty} \frac{(-1)^{k+1}}{2 k-1} \cos 2(2 k-1) x .
\end{aligned}
$$

Note that the sum of the absolute values of the Fourier coefficients is divergent. Thus, potentials with jump discontinuities must be excluded in a theory which uses the classical version of Gershgorin's theorem to estimate the eigenvalues of the matrix $A$ in Section 1. This shows the advantage of our $L^{2}$ approach. The eigenvalues corresponding to the potential $q_{3}(x)$ satisfy a transcendental equation involving only elementary functions, and the first 16 roots of this equation were obtained by using the ZERP algorithm due to Kahan. This algorithm gives upper and lower bounds for the roots and the eigenvalues were found with an accuracy of $10^{-8}$. The eigenvalues were also computed by the Rayleigh-Ritz procedure with the space of trial functions spanned by 60 sine functions, and differed from the correct ones by approximately $10^{-5}$. This indicates that our method for solving the inverse Sturm-Liouville problem will converge slowly for potentials with discontinuities. This is borne out in Table 4 . In the case where we use 16 eigenvalues and the dimension of the space of trial functions is 24 we find that the errors in all the Fourier coefficients are of order $10^{-4}$, except 
for the last where the error is $2.8 \cdot 10^{-3}$. The corresponding potential is given in Figure 1, and we note the Gibb's phenomenon.

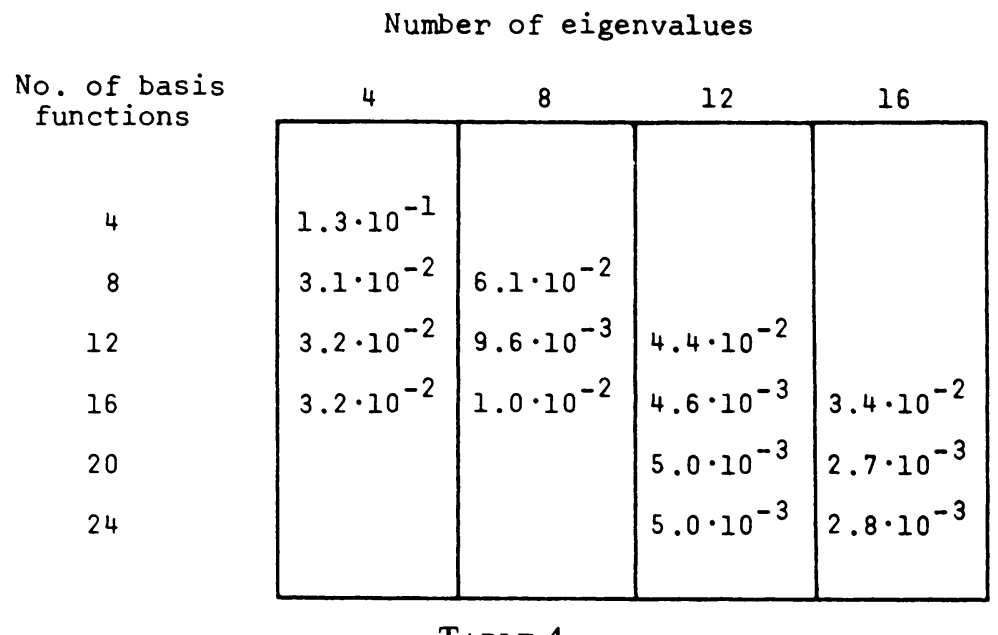

TABLE 4

Errors in the Fourier coefficients

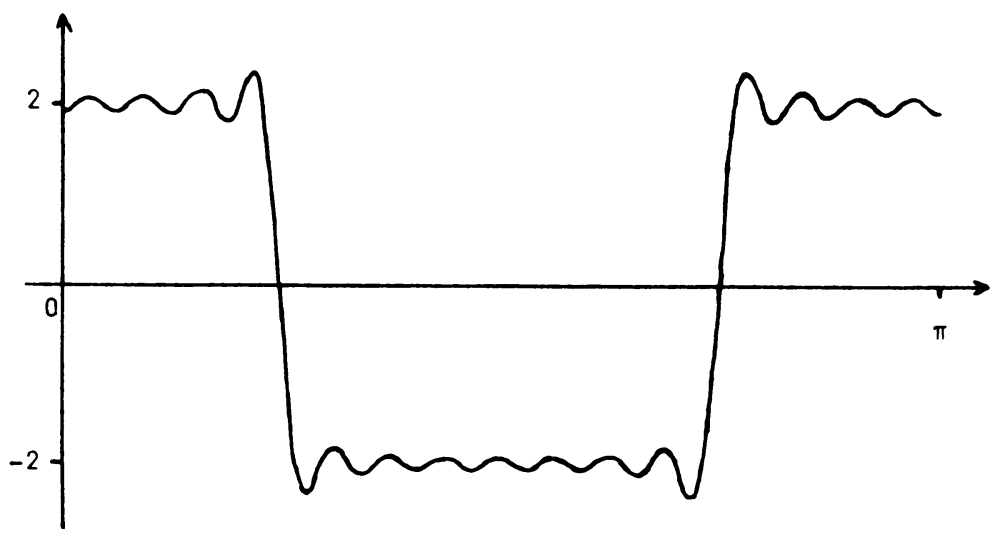

FIGURE 1

Discontinuous potential reconstructed from 16 eigenvalues

In our numerical experiments we have used Gaussian elimination to solve the systems $\left(A_{\nu}-\lambda_{\nu}\right) x=a_{\nu}$ and used 20 complete sweeps of the functional iterations (3.1). Clearly, this could be replaced by a more economical technique. However, our objective has been to present a method which works and can be proved to work. The method is only local and it is not clear if one should strive for an optimal implementation at this stage.

Acknowledgements. The numerical approach presented here was suggested to the author by Professor Olof B. Widlund. The research has been supported in part by the National Science Foundation under Grant No. MPS-75-10363. 
1. L. ANDERSON, "On the effective determination of the wave operator from given spectral data in the case of a difference equation corresponding to a Sturm-Liouville differential equation," J. Math. Anal. Appl., v. 29, 1970, pp. 467-497.

2. G. E. BACKUS \& J. F. GILBERT, "Numerical applications of a formalism for geophysical inverse problems," Geophys. J. Roy. Astronom. Soc., v. 13, 1967, pp. 247-276.

3. V. BARCILON, "Iterative solution of the inverse Sturm-Liouville problem," J. Mathematical Phys., v. 15, 1974, pp. 287-298.

4. G. BORG, "Eine Umkehrung der Sturm-Liouvilleschen Eigenwertaufgabe," Acta Math., v. 78, 1946, pp. $1-96$.

5. P. G. CIARLET, M. H. SCHULTZ \& R. S. VARGA, "Numerical methods of high-order accuracy for nonlinear boundary value problems. III. Eigenvalue problems," Numer. Math., v. 12, 1968, pp. 120-133.

6. E. CODDINGTON \& N. LEVINSON, Theory of Ordinary Differential Equations, McGrawHill, New York, 1955.

7. R. COURANT \& D. HILBERT, Methods of Mathematical Physics, Vol. 1, Interscience, New York, 1953.

8. S. FRIEDLAND, "Inverse eigenvalue problems," Linear Algebra and Appl., v. 17, 1977, pp. $15-51$.

9. F. R. GANTMACHER \& M. G. KREIN, Oszillationsmatrizen, Oszillationskerne und kleine Schwingungen Mechanischer Systeme, Akademie-Verlag, Berlin, 1960.

10. I. M. GEL'FAND \& B. M. LEVITAN, "On the determination of a differential equation from its spectral function," Amer. Math. Soc. Transl. (2), v. 1, 1955, pp. 253-304.

11. K. P. HADELER, "Ein Inverses Eigenwertproblem," Linear Algebra and Appl., v. 1, 1968, pp. 83-101.

12. O. H. HALD, On Discrete and Numerical Inverse Sturm-Liouville Problems, Ph.D. Thesis, New York Univ., New York, N. Y., 1972.

13. O. H. HALD, "The inverse Sturm-Liouville problem with symmetric potentials," Acta. Math. (To appear.)

14. G. H. HARDY, J. E. LITTLEWOOD \& G. PÓLYA, Inequalities, 2nd ed., Cambridge Univ. Press, London, 1952.

15. H. HOCHSTADT, "The inverse Sturm-Liouville problem," Comm. Pure Appl. Math., v. 26,1973 , pp. $715-729$.

16. H. HOCHSTADT, "Well-posed inverse spectral problems," Proc. Nat. Acad. Sci. U.S.A. v. 72, 1975, pp. 2496-2497.

17. T. KATO, "On the upper and lower bounds of eigenvalues," J. Phys. Soc. Japan, v. 4, 1949, pp. 334-339.

18. M. G. KREIN, "Solution of the inverse Sturm-Liouville problem," Dokl. Akad. Nauk SSSR (N. S.), v. 76, 1951 , pp. $21-24$.

19. M. G. KREIN, "Determination of the density of a non-homogeneous symmetric cord by its frequency spectrum," Dokl. Akad. Nauk SSSR (N. S.), v. 76, 1951, pp. 345-348.

20. F. LABORDE, "Sur un problème inverse d'un problème de valeurs propres," $C . R$. Acad. Sci. Paris Ser. A-B. v. 268, 1969, pp. A153-A156.

21. N. LEVINSON, "The inverse Sturm-Liouville problem," Mat. Tidsskr. B, 1949, pp. 25-30.

22. B. M. LEVITAN, "On the determination of a Sturm-Liouville equation by two spectra," Amer. Math. Soc. Transl. (2), v. 68, 1968, pp. 1-20.

23. V. A. MARČENKO, "Concerning the theory of a differential operator of the second order," Dokl. Akad. Nauk SSSR (N. S.), v. 72, 1950, pp. 457-460.

24. P. MOREL, "Des algorithmes pour le probleme inverse des valeurs propres," Linear Algebra and Appl., v. 13, 1976, pp. 251-273.

25. F. I. NIORDSON, "A method for solving inverse eigenvalue problems," in Recent Progress in Applied Mechanics, The Folke Odquist Volume (B. Broberg, J. Hult \& F. Niordson, Editors), Almquist \& Wiksell, Stockholm, 1967, pp. 375-382.

26. G. N. de OLIVEIRA, "Note on an inverse characteristic value problem," Numer. Math., v. 15,1970, pp. 345-347.

27. G. STRANG \& G. J. FIX, An Analysis of the Finite Element Method, Prentice-Hall, Englewood Cliffs, N. J., 1973.

28. Tables Relating to Mathieu Functions, National Bureau of Standards, Columbia Univ. Press, 1951. 